\title{
Background monitoring and spatial analysis of total organic carbon in groundwater in Qazvin plain
}

\author{
M. Panahi Fard ${ }^{1}$, AH. Mahvi², A. Bahojb ${ }^{3}$, S. Naderi ${ }^{3}, \underline{\text { H. Karyab }}^{1}$
}

\begin{abstract}
${ }^{1}$ Department of Environmental Health Engineering, School of Health, Qazvin University of Medical Sciences, Qazvin, Iran
${ }^{2}$ Department of Environmental Health Engineering, School of Health, Tehran University of Medical Sciences, Tehran, Iran

${ }^{3}$ Department of Environmental Health Engineering, Health Centre, Qazvin University of Medical Sciences, Qazvin, Iran
\end{abstract}

Corresponding Address: Hamid Karyab, Department of Environmental Health Engineering, Health Faculty, Qazvin University of Medical Sciences, Qazvin, Iran

Tel: +98-912-7830583; Email: hkaryab@qums.ac.ir

Received: 21 Feb 2018; Accepted: 15 May 2018

\section{Abstract}

Background: Total organic carbon (TOC) is an indicator which presents water pollution via wastewater and organic pollutants including pesticides. TOC content in water presents organic matter and resulted that some countries use TOC as an indicator for assessing row water quality and water treatment facilities.

Objective: This study was done to determine total organic carbon concentration in groundwater resources in Qazvin plain.

Methods: Eighty-one water samples were selected randomly in arid and semi-arid climates in Qazvin plain in winter and spring of 2016. Sample stations were located in Qazvin, Takestan, Abyek and Buin Zahra cities. TOC analysis was done with SGE ANATOC ${ }^{\text {TM }}$ Series II analyzer.

Findings: The average of TOC concentrations were detected $0.88,0.68,0.81$ and $0.79 \mathrm{mg} / \mathrm{L}$ in Qazvin, Takestan, Abyek and Boin Zahra cities, respectively, with the mean of $0.79 \mathrm{mg} / \mathrm{L}$. In addition, no significant relationship was found between land use and TOC concentration in groundwater.

Conclusion: The average of detected TOC in groundwater resources in the study area was lower than acceptable levels provided by the United States Environmental Protection Agency. The obtained results revealed that there were low potential for production of disinfection by-products in chlorination process. Low levels of TOC in water resources can be due to the performance of filtration and reduction processes.

Keywords: Total organic carbon, Qazvin plain, Drinking water resources

Citation: Panahi Fard M, Mahvi AH, Bahojb A, Naderi S, Karyab H. Background monitoring and spatial analysis of total organic carbon in groundwater in Qazvin plain. J Qazvin Univ Med Sci 2018; 22(2): 20-27. 


\title{
يايش زمينه و نقشه يراكندَّى كل كربن آلى (TOC) در منابع آب شرب زيرزمينى دشت قزوين
}

\author{
مسعود پيناهى فرد'، دكتر اميرحسين محوى '، اكرم باحجب"، شيرين نادرى '، دكتر حميد كارياب'
}

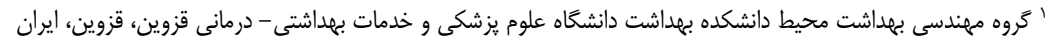

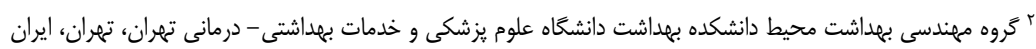

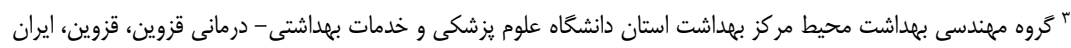

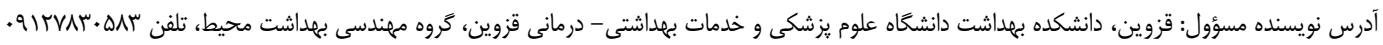

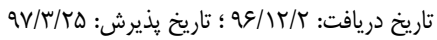

زمينه: كل كربن آلى (Total organic carbon; TOC) شاخصى است كه نشاندهنده احتمال آلودگى آب به فاضلاب و آلايندهاى آلى نظيـر

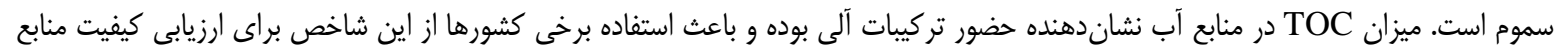
آب خام و سيستمهاى تصفيه آب شده است.

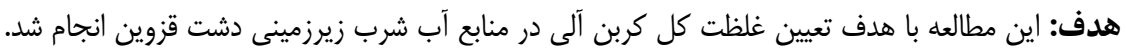

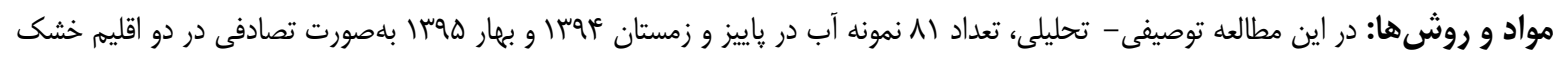

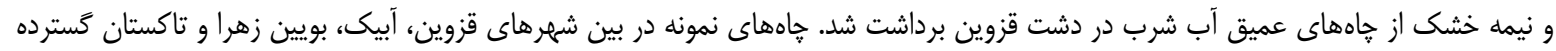

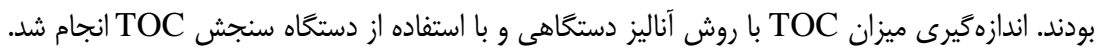

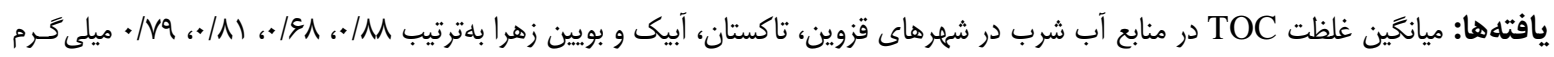

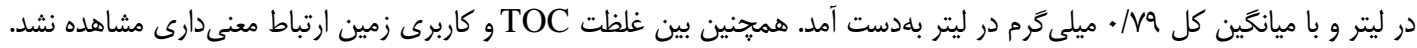

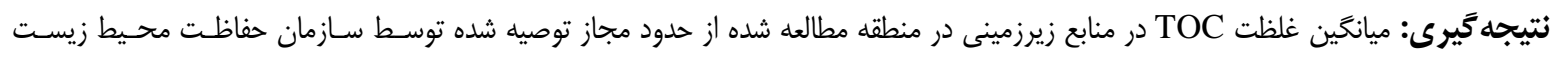

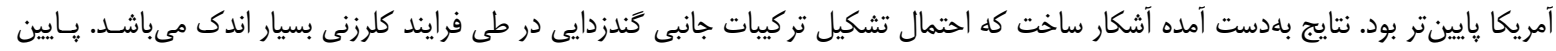

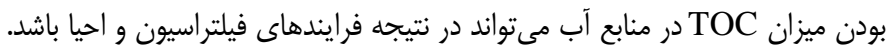

كليدوازهها: كل كربن آلى، دشت قزوين، منابع آب شرب

\section{مقدمه:}

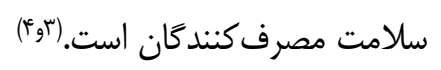

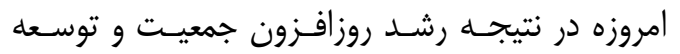

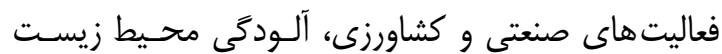

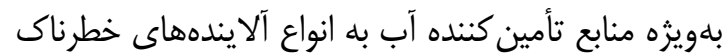

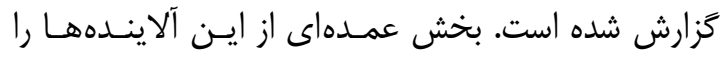

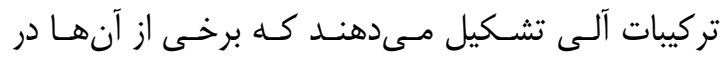

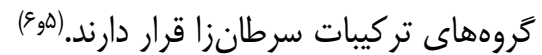

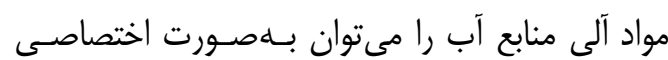

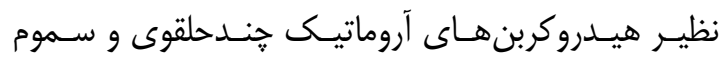

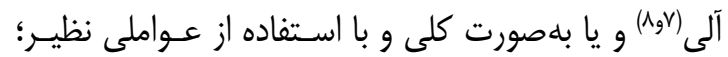

آبهاى زيرزمينى از منـابع حيـاتى تـأمين آب بـراى

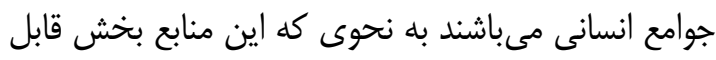

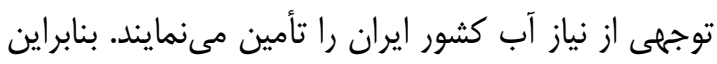

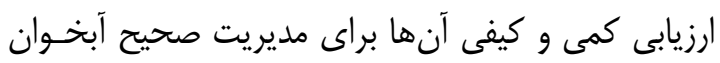

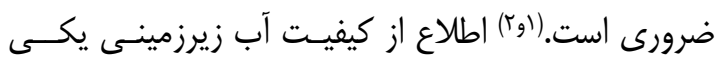

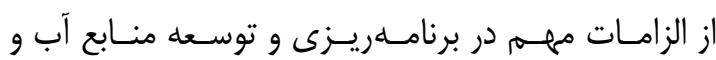

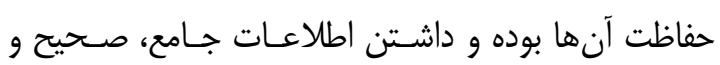

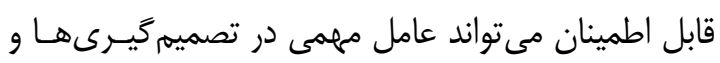

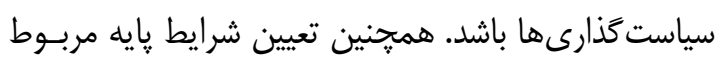

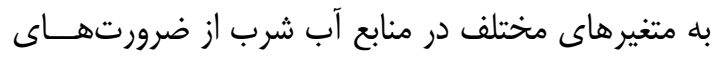


طراحى و مطابق آن تصفيهخانههـاى موجـود توسـعه داده

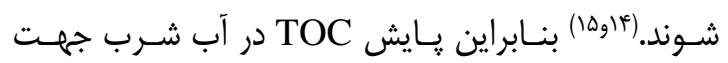
مديريت خطرات سلامتى ضرورى است. در خصوص حد مجـاز TOC در آب شـرب، اسـتاندارد ملى وجود ندارد. ضمن آن كه سازمان بهداشت جهانى نيـز

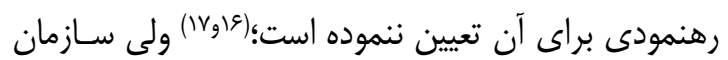
حفاظت محيط زيست آمريكا حداكثر ميـزان مجـاز

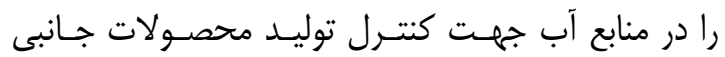
كَندزدايى مثل ترى هالومتانها به ميزان أ ميلـى

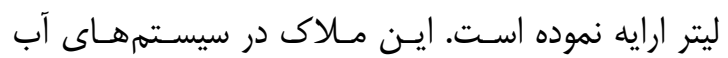

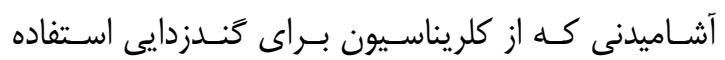

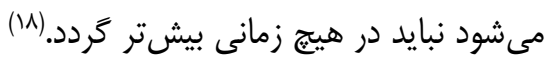

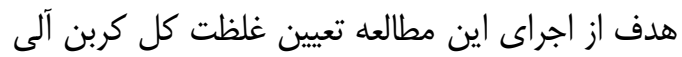

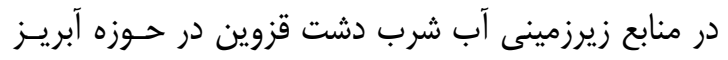

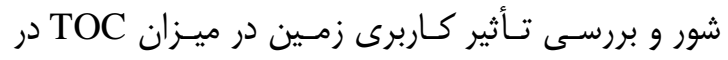

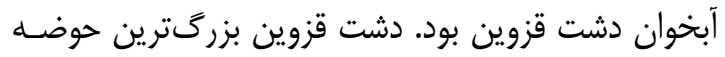

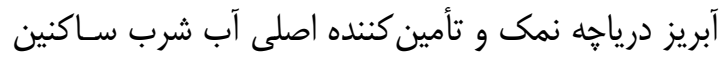
دشت مىباشد بلنحوى كه سهم بهرهبردارى براى تـأمين

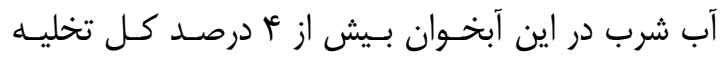

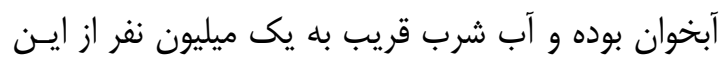
دشت تأمين مى

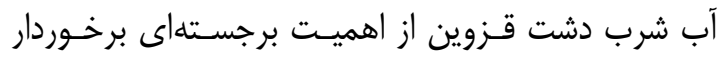

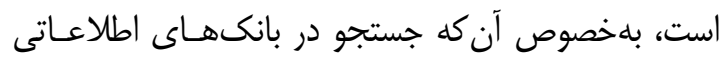
نشان داد مطالعه مشابهى جهت پايش TOC Tـ در آبخـوان

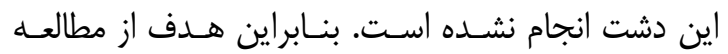

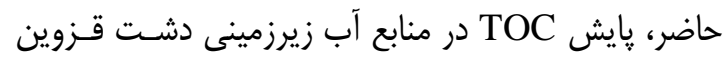

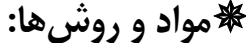

اين مطالعه توصيفى در طى فصـول يـاييز و زمسـتان

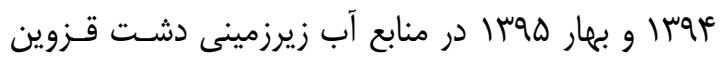

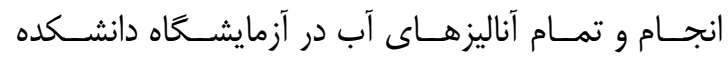

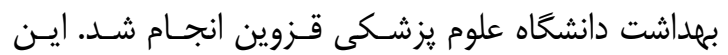

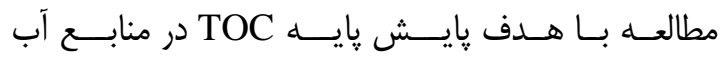

كل كربن آلى (TOC)، اكسيثرن مـورد نيـاز بيوشـيميايى

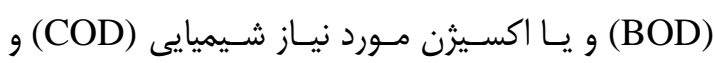
كربن آلى محلول (DOC) اندازهخيرى نمود. (a) (a)

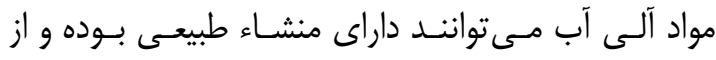

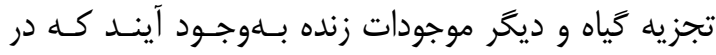

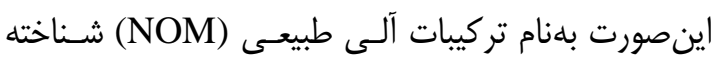

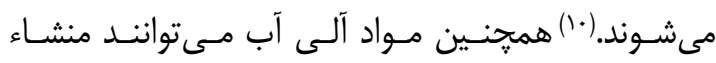

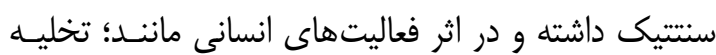

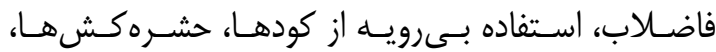

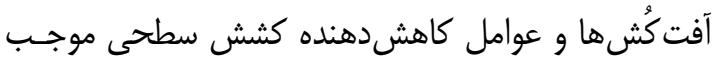

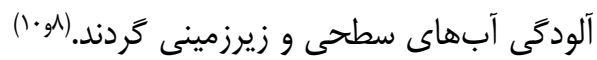

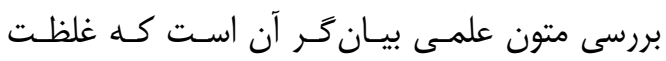
تركيبات آلى شناسايى شده در منـابع آب زيرزمينسى غالبـ استـاً

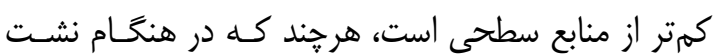

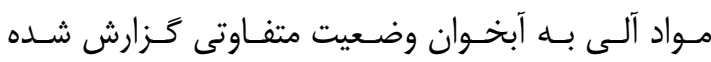

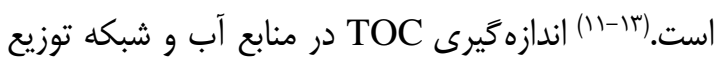

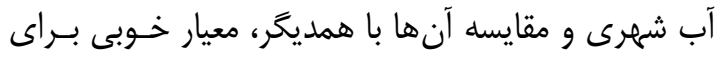

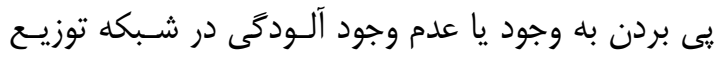

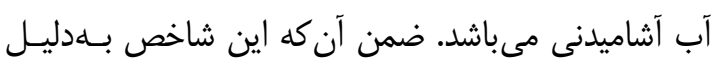

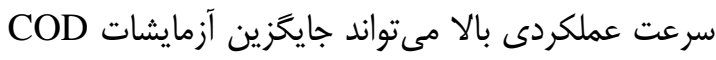

$$
\text { و }
$$

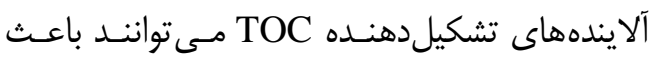

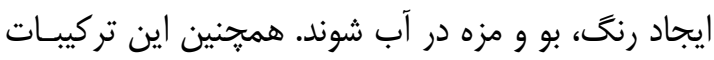

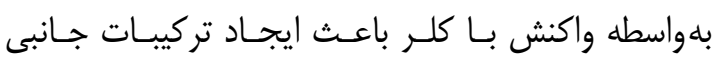

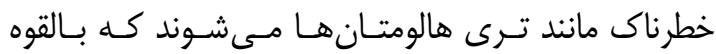

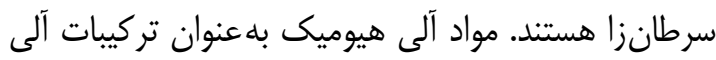

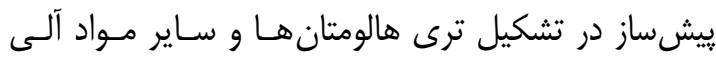
هالوزنه نقش دارند. طى سال هاى اخير در اكثر كشورهاى تهاي

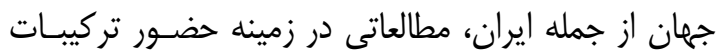

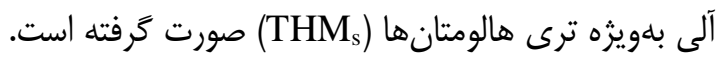

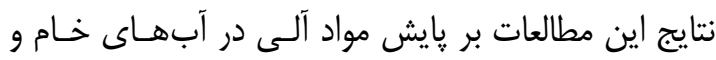

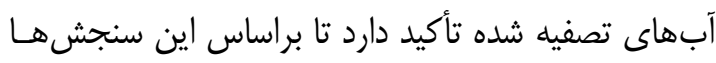

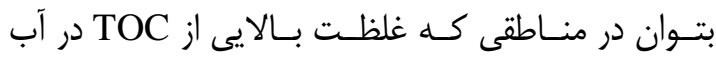

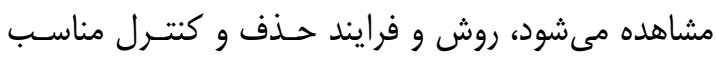




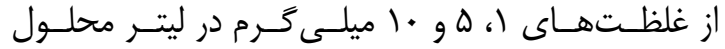

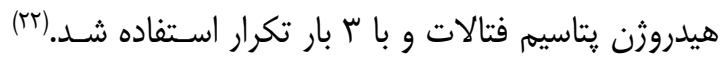

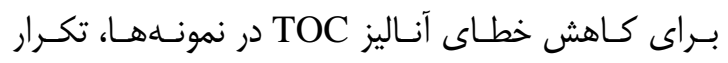

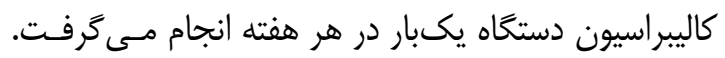

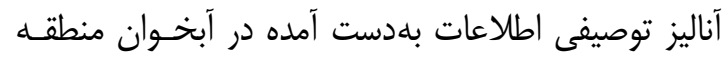

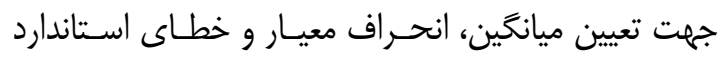

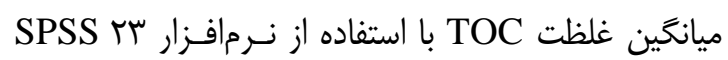

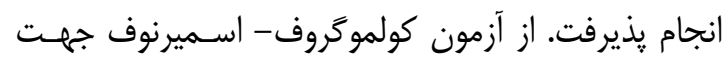

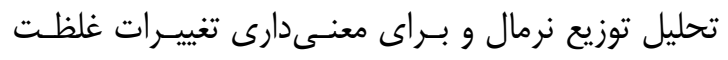

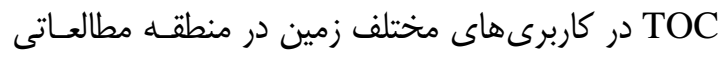

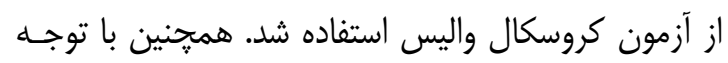

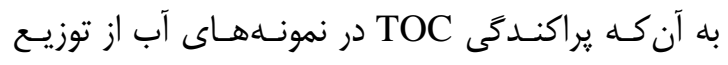

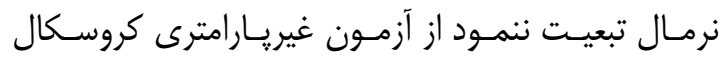

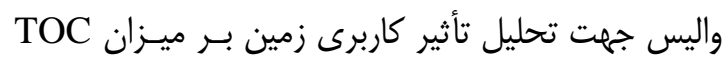
منابع زيرزمينى استفاده شد.

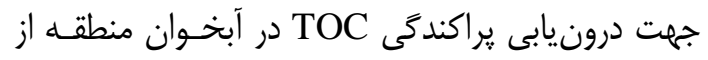

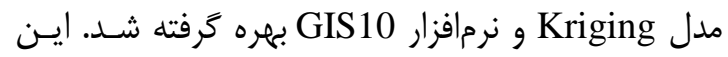
مدل قادر است كه نسبت به ساير روشها با با قابليت بالايى

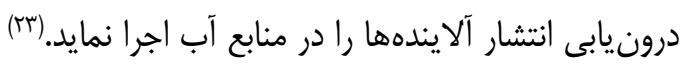

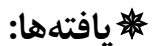

ميانكين غلظت TOC در منابع تأمين آب شرب واقع

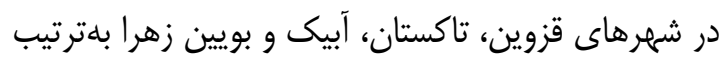
غل غلظت در كل منطقه /V9/ • ميلى گرم در ليتر بـود. بيشـينه

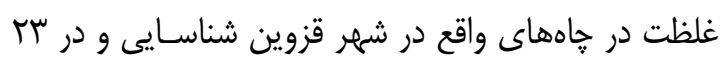

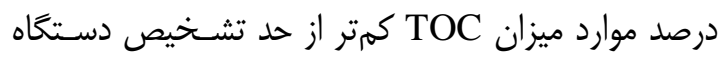

$$
\text { (1/ ميلى گرم در ليتر) حاصل شد. }
$$

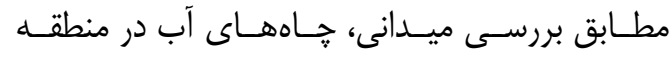

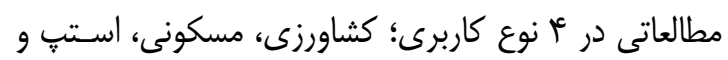
مختلط قرار داشتند. مقادير غلظت شناسـايى شـده در كاربرىهاى مختلف زمين در جدول شماره ا ارايه شده

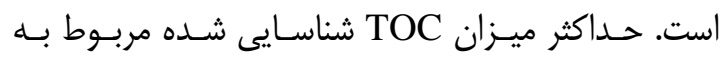
جاههاى واقع در مناطق مسكونى بود.
زيرزمينى در حوضه آبريز شور در محـدوده دشـت قـزوين

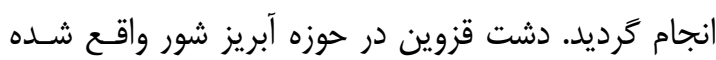

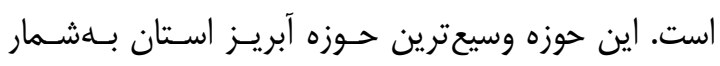

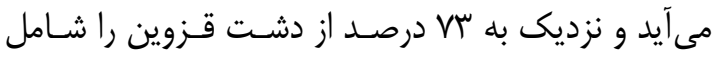

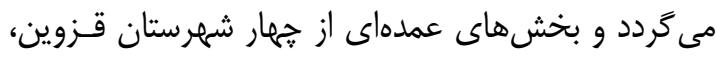

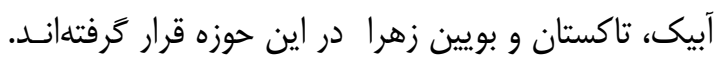

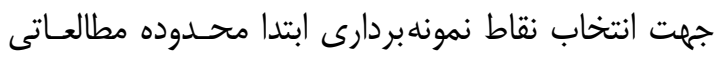
با توجه به تقسيمات كشورى به مناطق مختلف تقسيميم و

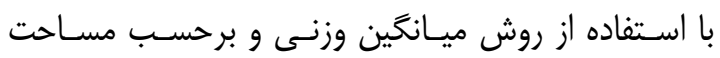

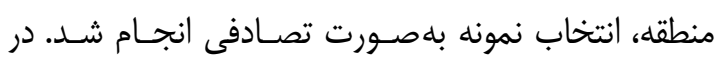

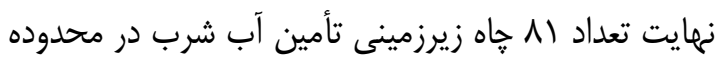

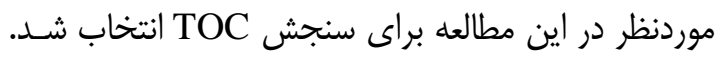

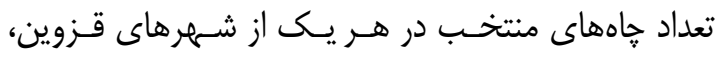

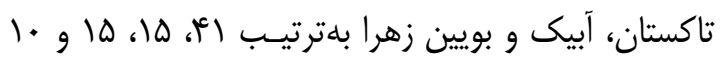

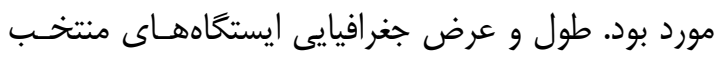

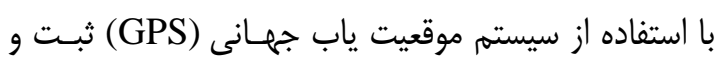
در نرمافزار سيسته اطلاعـات جغرافيـيـ (ArcGIS) وارد

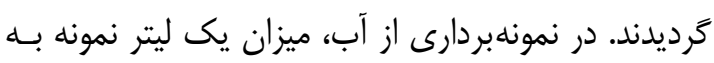
روش ساده (grab) در ظروف شيشهاى كه قبلاً با اسيد و

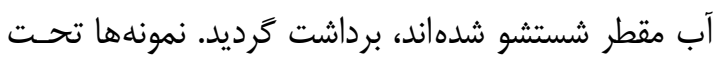

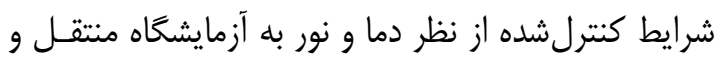

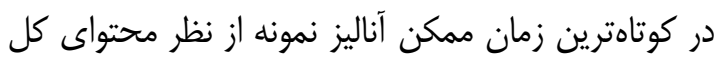

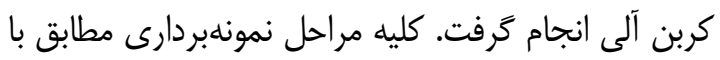

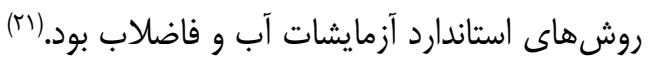
اندازهخيرى ميزان TOC با روش آناليز دسـتخاهى و بـانـا SGE ANATOCTM Series II استفاده از دستخاه آنـاليز

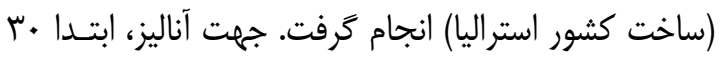

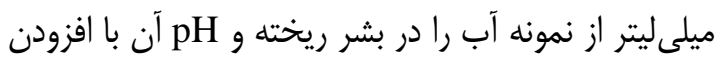

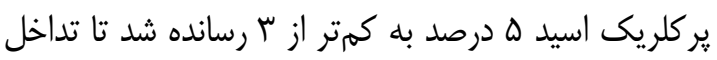

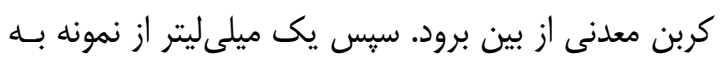

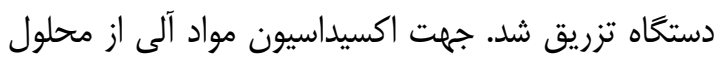

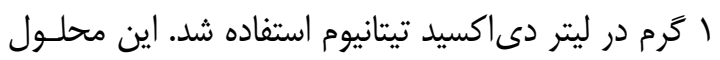

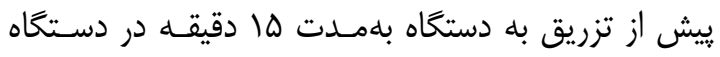
اولتراسونيك يكنواخت و جهت ترسيم منحنى كاليبراسيون 
بر روى كل كـربن آلى، كـربن آلى ذرهاى، كـربن آلى

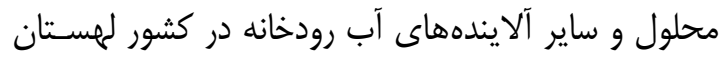

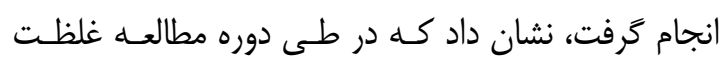

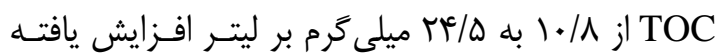

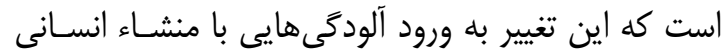
به اين رودخانه نسبت داده شد.

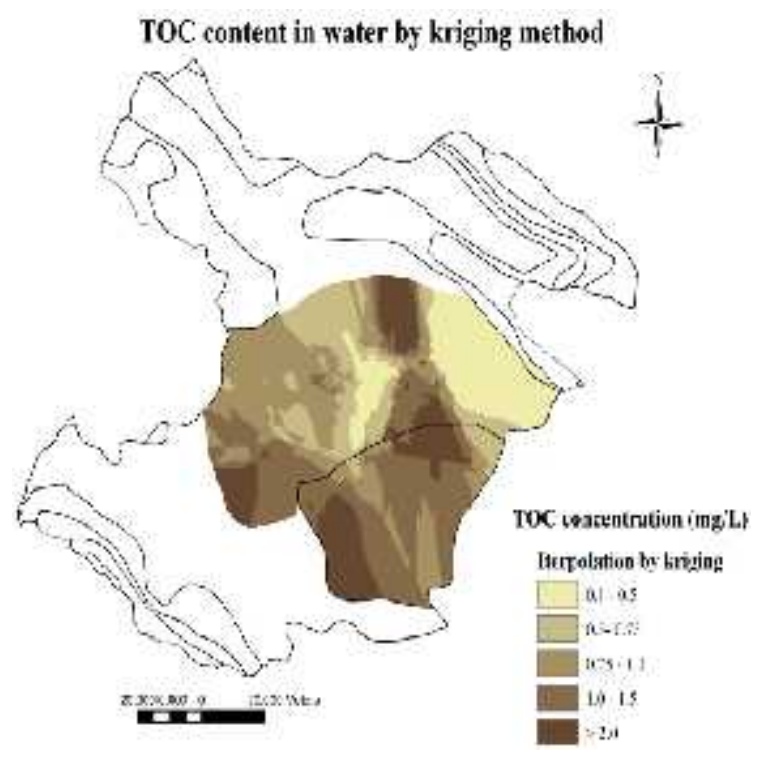

شكل ا - توزيع يراكندَّى غلظت TOC در منابع آب زيرزمينى در منطقه مطالعاتى در دشت قزوين

نتايج آزمون كروسـكال والـيس فرضـيه تأثيريـذيرى

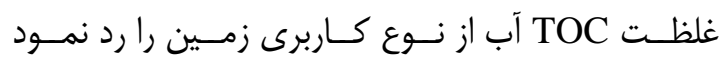
(P=0.27 F=4 chi2=5.12)

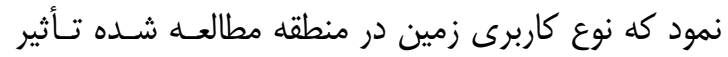

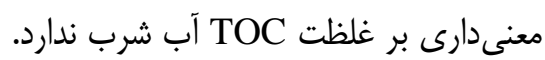
اطلاعات منابع آب آشاميدنى در ايالات متحده آمريكا

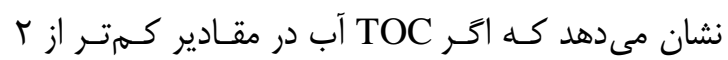
ميلى گرم در ليتر در خروجـى آب آشـاميدنى حفـظ گـردد، احتمال زيادى وجود دارد كه مقادير THMs بيش از ميزان

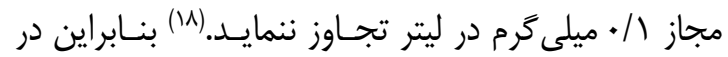

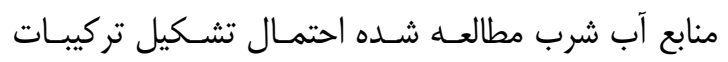

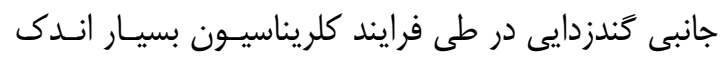

همان گونه كه در شـكل شـماره ا نمـايش داده شــه

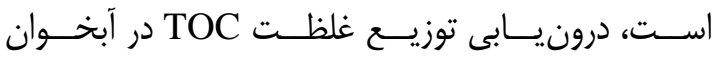

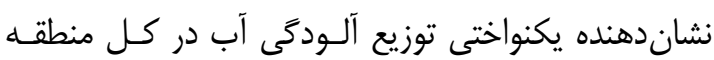

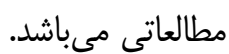

جدول ا - آناليز توصيفى غلظت TOC در آب شرب در مخر كاربرى جاى مختلف در منطقه مطالعاتى (ميلى ترم در ليتر)

\begin{tabular}{|c|c|c|c|c|}
\hline حد اطمينان & بيشينه & خطاى استدارد & انحراف معيار \ميانكين & كاربرى \\
\hline$. / 4-. / 94$ & $r / r$ & 每 & $\cdot|9 V \pm \cdot / V|$ & كشاورزى \\
\hline$\cdot / V^{c}-1 / 1 \Lambda$ & $4 / 1$ & .111 & $. / 94 \pm . / V^{c}$ & مسكونى \\
\hline$\cdot / 1 Q-1 / 1 \Lambda$ & r & תוא. & $\cdot / 9 V \pm \cdot / V V$ & استب \\
\hline$\cdot / 4 \mid-1 / \cdot 0$ & $r / \mu$ & .110 & $\cdot / v \Psi \pm \cdot / 9 q$ & مختلط \\
\hline
\end{tabular}

\section{من بحث و نتيجه}

ميانگين و بيشينه غلظت كربن آلى دئل در منابع زيرزمينى

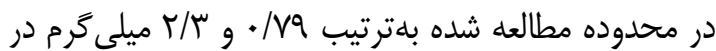

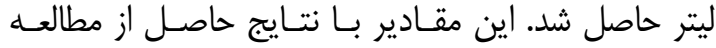

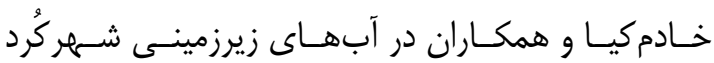

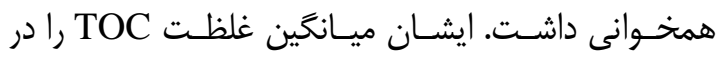
جاههاى بررسى شده به ميزان ץ.

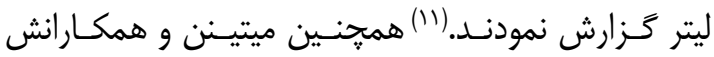

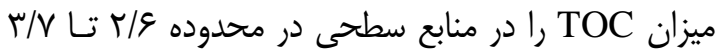

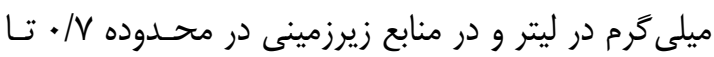

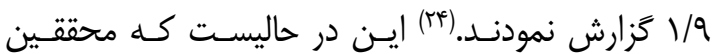
ديخرى بيشينه غلظت TOC را در منابع آبهاى سـطحى تا لأ ميلى گرم در ليتر نيز گزارش نمودهاند.

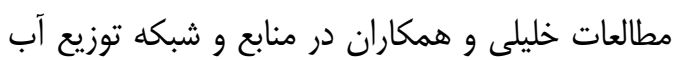
شرب شهر گركان نشان داد كه ميانكين غلظت TOC در

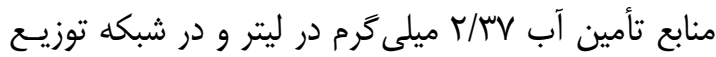

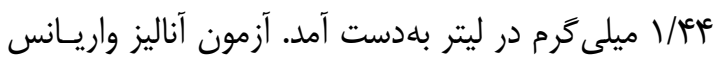

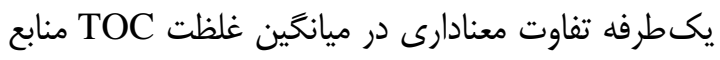

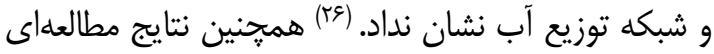
كه توسط سييك و همكاران در سالهاى 1991 تـا 1999 
https://www.civilica.com/Paper-ICWR01ICWR01_178.html. [In Persian]

3. Sener S, Sener E, Davraz A. Assessment of groundwater quality and health risk in drinking water basin using GIS. J Water Health 2017; 15(1): 112-32. doi: 10.2166/wh.2016.148.

4. Chabukdhara M, Gupta SK, Kotecha Y, Nema AK. Groundwater quality in Ghaziabad district, Uttar Pradesh, India: multivariate and health risk assessment. Chemosphere 2017; 179: 167-78. doi: 10.1016/j.chemosphere.2017.03.086.

5. Karyab H, Mahvi AH, Nazmara S, Bahojb A. Determination of water sources contamination to diazinon and malathion and spatial pollution patterns in Qazvin, Iran. Bull Environ Contam Toxicol 2013; 90(1): 12631. doi: 10.1007/s00128-012-0880-8.

6. Karyab H, Nasseri S, Ahmadkhaniha R, Rastkari N, Mahvi AH, Nabizadeh R, et al. Determination and source identification of polycyclic aromatics hydrocarbons in Karaj River, Iran. Bull Environ Contam Toxicol 2014; 92(1): 50-6. doi: 10.1007/s00128-0131140-2.

7. Singh DK, Kawamura K, Yanase A, Barrie LA. Distributions of polycyclic aromatic hydrocarbons, aromatic ketones, carboxylic acids, and trace metals in arctic aerosols: long-range atmospheric transport, photochemical degradation/production at polar sunrise. Environ Sci Technol 2017; 51(16): 8992-9004. doi: 10.1021/acs.est. $7 b 01644$.

8. Rousis NI, Bade R, Bijlsma L, Zuccato E, Sancho JV, Hernandez F, et al. Monitoring a large number of pesticides and transformation products in water samples from Spain and Italy. Environ Res 2017; 156: 31-8. doi: 10.1016/j.envres.2017.03.013.
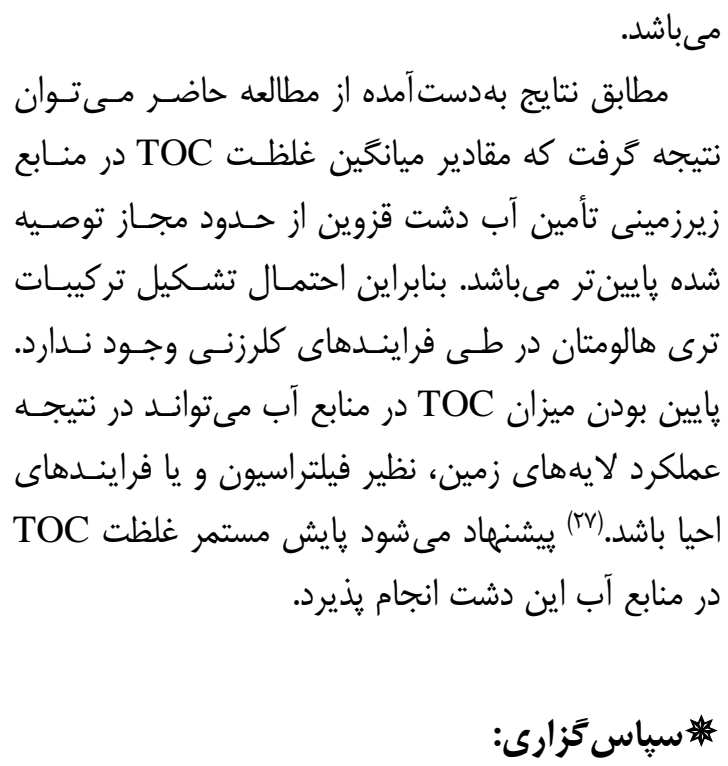

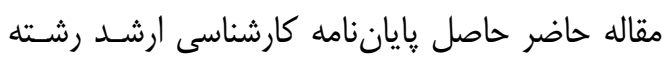

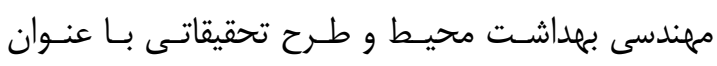

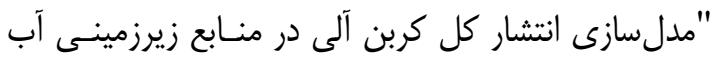

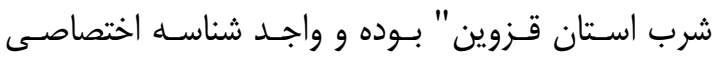

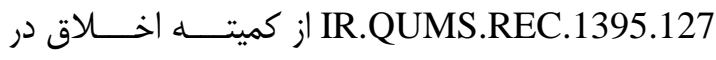
يزوهش هاى زيست يزشكى دانشكاه علوم يزشكى قزوين

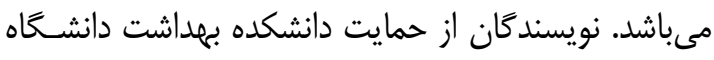

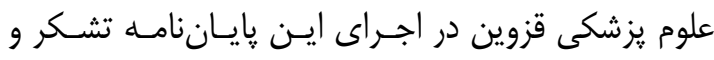
قدردانى مىنمايند.

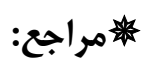

1. Peng TR, Huang C, Chen J, Zhan WJ, Chiang LW, Chang LC. Evaluating the relative importance of groundwater recharge sources in a subtropical alluvial plain using tracer-based ternary End Member Mixing Analysis (EMMA). Water Resources Management 2016; 30(11): 3861-78.

2. Askari M, Mosaedi A, Dehghani AA, Meftah halghi M. Application of geostatistics and Gis analysis, in study of groundwater quality spatial variability, case study in Qazvin plain. International Conference in Water Resources 2009; Available at: 
9. Christian E, Batista JR, Gerrity D. Use of COD, TOC, and fluorescence spectroscopy to estimate BOD in wastewater. Water Environ Res 2017; 89(2): 168-77. doi: 10.2175/ 106143016X14504669768976.

10. Hidayah EN, Chou YC, Yeh HH. Characterization and removal of natural organic matter from slow sand filter effluent followed by alum coagulation. Appl Water Sci 2018; 8(1): 3 .

11. Khademikia S, Rafiee Z, Amin MM, Poursafa P, Mansourian M, Modaberi A. Association of nitrate, nitrite, and total organic carbon (TOC) in drinking water and gastrointestinal disease. J Environ Public Health 2013. doi: 10.1155/2013/603468.

12. Siepak J. Total organic carbon (TOC) as a sum parameter of water pollution in selected Polish rivers (Vistula, Odra, and Warta). CLEAN-Soil Air Water 1999; 27(5): 282-5. doi: 10.1002/(SICI)1521-401X(199911)27:5< 282:AID-AHEH282>3.0.CO;2-2.

13. Lapworth DJ, Baran N, Stuart ME, Ward RS. Emerging organic contaminants in groundwater: a review of sources, fate and occurrence. Environ Pollut 2012; 163: 287303. doi: 10.1016/j.envpol.2011.12.034.

14. Dubber D, Gray NF. Replacement of chemical oxygen demand (COD) with total organic carbon (TOC) for monitoring wastewater treatment performance to minimize disposal of toxic analytical waste. J Environ Sci Health A Tox Hazard Subst Environ Eng 2010; 45(12): 1595-600. doi: 10.1080/10934529.2010.506116.

15. Postigo C, Richardson SD, Barcelo D. Formation of iodo-trihalomethanes, iodohaloacetic acids, and haloacetaldehydes during chlorination and chloramination of iodine containing waters in laboratory controlled reactions. J Environ Sci (China)
2017; 58: 127-34. doi: 10.1016/j.jes.2017.04. 009.

16. ISIRI, Drinking water - Physical and chemical specifications, 1053, 5th.revision, Institute of Standards and Industrial Research of Iran 2010 Available at: nmbk.ir/ harticle/nmbk-1392-2-22-10-51-371053.pdf.

[In Persian]

17. WHO. Guidelines for drinking-water quality. 4th ed. World Health Organization; 2011 Available at: http://www.who.int/ water_sanitation_health/publications/gdwq41st-addendum/en/.

18. USEPA. Drinking water guidance on disinfection by-products. https://www.epa.ie/ pubs/advice/drinkingwater/DrinkingWaterGui de4_v8.pdf. Accessed in: 2014.

19. Hajimirmohammad Ali RS, Karyab H. Predicting nitrate concentration in groundwater resources using lumpedparameter model: case study in Qazvin plain. Iran J Health Environ 2016; 8(4): 459-70. [In Persian]

20. Barikani E, Ahmadian M, Khalilian S. Sustainable operation of groundwater resources in the agricultural sector: case study of Qazvin plain subsection. J Agricultural Economics and Development 2011; 25(2): 253-62. Available at: https://www.civilica. com/Paper-JR_JEAD-JR_JEAD-25-2_013. html [In Persian]

21. Rice ED, Baird RB, Eaton AD. Standard methods for the examination of water and wastewater. 5th ed. New York: American Public Health Association, American Water Works Association, Water Environment Federation; 2005.

22. Karyab H, Karyab F, Haji-Mirmohammad Ali R. Optimization of adsorption conditions for removal of total organic carbon from drinking water using polypropylene and 
titanium dioxide nano-composite by response surface methodology. Desalination and Water Treatment 2017; 98: 144-51. doi: 10.5004/ dwt.2017.21670.

23. Murphy RR, Curriero FC, Ball WP. Comparison of spatial interpolation methods for water quality evaluation in the Chesapeake Bay. J Environ Eng 2010; 136(2): 160-71. doi: 10.1061/(ASCE)EE. 1943-7870.0000121.

24. Miettinen IT, Vartiainen T, Martikainen PJ. Phosphorus and bacterial growth in drinking water. Appl Environ Microbiol 1997; 63(8): 3242-5.

25. Kortelainen P. Content of total organic carbon in Finnish lakes and its relationship to catchment characteristics. Can J Fish Aquat Sci 1993; 50(7): 1477-83. doi: 10.1139/f93168.

26. Khalili KH. The survey of TOC and NO3 contents in Gorgan drinking water sources and Distribution system-district. 2010; Islamic azad university. Available at: srbiau.ac.ir/Files/environment/24906.pdf. [In Persian]

27. Keesstra SD, Geissen V, Mosse K, Piiranen S, Scudiero E, Leistra M, et al. Soil as a filter for groundwater quality. Curr Opin Environ Sustain 2012; 4(5): 507-16. doi: 10. 1016/j.cosust. 2012.10.007. 